\title{
Optimization of Radial Magnetorheological Valves with Constraints of Volume, Pressure Drop and Power Consumption
}

\author{
Sofia Lydia Ntella*§, Minh-Trung Duong*§, Pooneh Mohaghegh*, Yoan Civet*, Zoltan Pataky** and \\ Yves Perriard* \\ * Integrated Systems Laboratory, EPFL, Neuchâtel, Switzerland \\ ** Division of Endocrinology, Diabetology, Nutrition and Therapeutic Education, Geneva University Hospitals, Switzerland

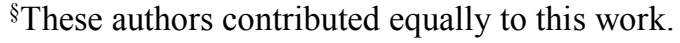

\begin{abstract}
Magnetorheological (MR) fluids are materials that consist of dispersions of ferromagnetic particles in a liquid carrier medium. Their tunable rheological properties under the application of an external magnetic field classify them in the category of smart materials. These properties are exploited for the creation of magnetorheological valves that act as actuators, dampers or shock absorbers. In this work, the optimal design of a miniature radial magnetorheological valve is presented. The target use of the valve is a wearable medical application, thus, constraints emerge regarding its size, pressure drop and power consumption. The optimization process relies on the Design of Experiments (DoE) techniques and the Response Surface Method (RSM). The optimal model is compared with the results of previous studies on different valve configurations.

Index Terms-Design of experiments, miniature magnetorheological valves, optimization, wearable device
\end{abstract}

\section{INTRODUCTION}

MR fluids are smart fluids with rheological properties that can change rapidly and reversibly. They consist of ferromagnetic micrometer-sized particles that are dispersed in a liquid carrier medium, such oil [1]. The application of an external magnetic field on the fluid domain brings about the magnetization of the particles and their alignment in parallel with the lines of the magnetic field. As a result, new structures, in the form of chains and opposed to the fluid's flow, are created, as in Fig. 1. The chain formation leads to the alteration of the rheology of the MR fluid to a semi-solid state [2]. On the contrary, in the absence of a magnetic field, the particles are dispersed randomly in the fluid and the MR fluid remains in its liquid state [3].

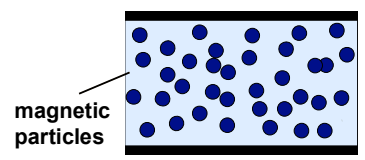

No magnetic field

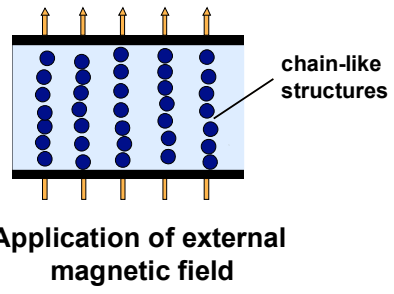

Fig. 1. MR fluid structure: particles dispersed in liquid, forming chains in case an external magnetic field is applied.

The MR effect is widely used, among others, in

This work was supported by the BRIDGE funding programme. mechanical systems such as shock absorbers, brakes, or clutches [4], [5], in haptics [6] as well as in biomedical applications [7]. More specifically, the MR valve is the fundamental component of an MR device and the most frequently used MR working mode. The valves consist of the following parts, as depicted in Fig. 2: (i) an inner and an outer iron core (ii) a coil, wound around the inner core (iii) a gap between the inner and the outer core. The MR fluid flows in the gap, and when a current is applied on the coil, a magnetic field, perpendicular to the fluid flow, is created and the viscosity of the MR fluid changes. The regions where the MR effect takes place are called active regions. The valve can be found in annular or radial configuration (Fig. 2), depending on the structure of the inner and outer core that affects the fluid flow path.

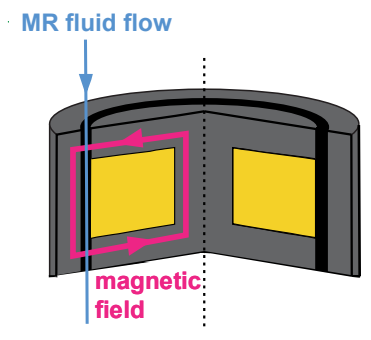

(a)

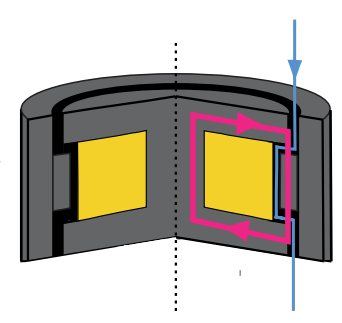

(b)
Fig. 2. Cross-section of (a) an annular and (b) a radial MR valve.

In the present work, we aim to achieve the optimal design of a miniature MR device, deployed in a wearable medical application, which is in the form of an insole (Fig. 3). The application is targeted at the prevention of diabetic foot ulcerations. More specifically, diabetic patients tend to present higher plantar pressure than the healthy population [8], in combination with peripheral neuropathy, a disorder that results in the loss of pain sensation [9]. As a consequence, ulcers are created on the plantar surface that can easily deteriorate and end up to a gangrene and an amputation. A first version of MR devices for this purpose, which act as pressure limiters has been designed [10], while the annular model of this version has already been optimized in our previous studies [11]. The pressure limiters appear in two states, the on-state, when the valve's coil is fed with current and the plantar load is sustained, and the off-state, when no 
current exists and the off-loading takes place. Each insole consists of a number of pressure limiters placed on the insole, as in Fig. 3. Every limiter consists of the valve, the variable-stiffness cushion used for offloading and the MR fluid that flows through the valve in the two chambers of the pressure limiter.

The goal of this work is to optimize the design of a radial MR valve and compare it with the existing designs. The optimal model is obtained considering the wearable application, in which the modules will be used, where volume and power consumption minimization seem imperative for two reasons: first of all, the development of an easy-to-use and comfortable device and, secondly, in order to obtain a good balance with regards to the charging frequency. At the same time, the pressure drop in each module affects decisively the load that each pressure limiter and the device in total can sustain, as explained in the next sections. Our methodology combines the DoE techniques and the RSM, together with a finite element model of the valve, constructed using Flux 2018.1.

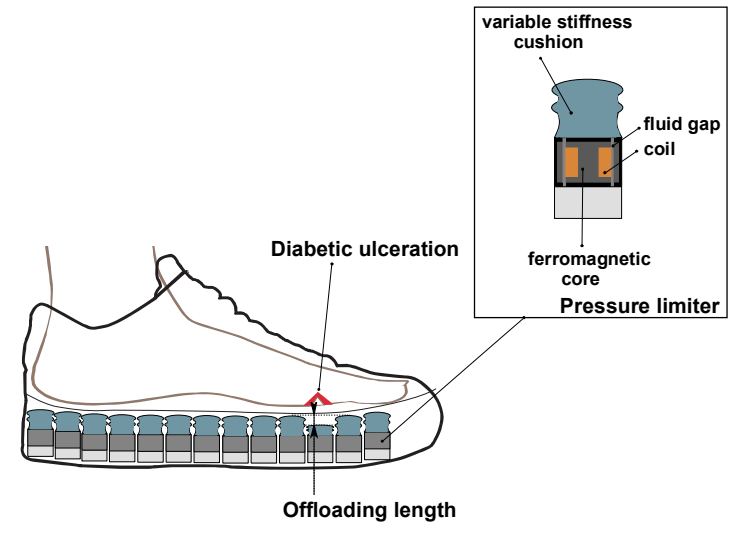

Fig. 3. Insole for plantar pressure offloading and diabetic ulceration prevention using MR pressure limiters.

\section{DESIGN OPTIMIZATION}

\section{A. Design Parameters}

The first step of the optimization process is to define the parameters that affect significantly the optimal model. These are the current density $J$ traversing the coil, which is responsible for the creation of the magnetic field, the total height of the valve $h_{t}$, the air gap length $g$, the radius of the inner core $r_{e}$, the difference $\Delta R=r_{e}-r_{i}$, where $r_{i}$ is the internal radius of the outer core, as well as the inner radius of the coil $r_{c}$. The geometrical parameters of the radial valve are depicted graphically in Fig. 4. Table I includes the range of the above parameters. According to our previous study [11], the ratio $h_{c} / h_{t}$, where $h_{c}$ the height of the coil, does not have a significant effect on the optimal model and, thus, it is not taken into account as an optimization factor. The outer core external radius of the valve $r_{o}$ is set to the constant value $5.5 \mathrm{~mm}$, in order to meet the constraints for sufficient pressure sensing resolution and system complexity [11].

Moreover, we have to define qualitatively and quantitatively, if possible, the optimization objectives that were described in the previous section. The total volume of the radial valve $V$ has to be minimized, as well as the power consumption $P$ of each valve, respecting the geometrical and current density requirements. The valve's pressure drop $\Delta \mathrm{P}$ has to be maximized with a minimum value of $700 \mathrm{kPa}$. This value derives from the observed plantar pressure values for healthy subjects, which lie in the range $350 \mathrm{kPa}$ to $400 \mathrm{kPa}$ [12], and which has to be sustained by our pressure limiter. Respectively, diabetic patients plantar pressure range from $700 \mathrm{kPa}$ to $1 \mathrm{MPa}$ for the same plantar area [13]. Thus, taking into account the safety range and the manufacturing tolerance, we set the minimum pressure drop to $700 \mathrm{kPa}$, higher than the value of $400 \mathrm{kPa}$.

TABLE I

DOE OPTIMIZATION FACTORS

\begin{tabular}{|c|c|c|c|c|}
\hline \multicolumn{2}{|c|}{ Factor } & Low & High \\
\hline 1 & Current density $\left(\mathrm{A} / \mathrm{mm}^{2}\right)$ & $\mathrm{J}$ & 9 & 15 \\
\hline 2 & Total height $(\mathrm{mm})$ & $\mathrm{h}_{\mathrm{t}}$ & 4 & 5 \\
\hline 3 & Airgap $(\mathrm{mm})$ & $\mathrm{g}$ & 0.15 & 0.25 \\
\hline 4 & Inner core radius $(\mathrm{mm})$ & $\mathrm{r}_{\mathrm{e}}$ & 4.2 & 4.8 \\
\hline 5 & $\mathrm{r}_{\mathrm{e}}-\mathrm{r}_{\mathrm{i}}(\mathrm{mm})$ & $\Delta \mathrm{R}$ & 0.6 & 0.8 \\
\hline 6 & Coil inner radius $(\mathrm{mm})$ & $\mathrm{r}_{\mathrm{c}}$ & 1.4 & 1.6 \\
\hline 7 & $\mathrm{~h}_{\mathrm{c}} / \mathrm{h}_{\mathrm{t}}$ & \multicolumn{3}{|c|}{0.5} \\
\hline 8 & Outer core external radius $(\mathrm{mm})$ & $\mathrm{r}_{\mathrm{o}}$ & \multicolumn{3}{|c|}{5.5} \\
\hline
\end{tabular}

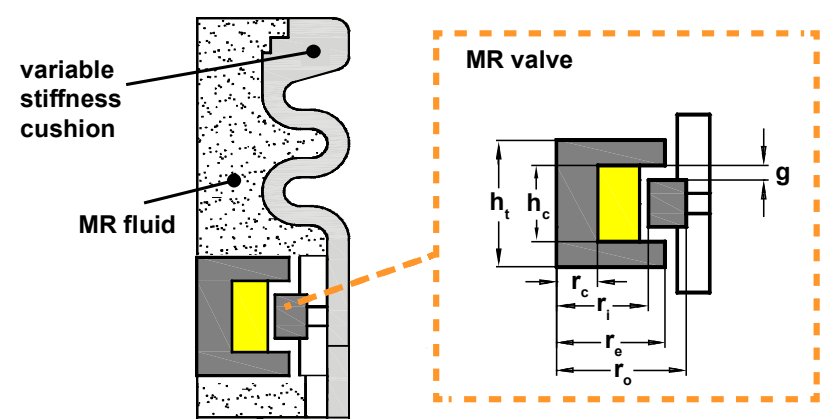

Fig. 4. Axis-symmetric cross-section and geometric model of a radial MR valve.

\section{B. Modeling of Valves}

First of all, the magnetic flux density $B$ in the gap was simulated using Flux 2018.1. The properties of the MRF132DG fluid by Lord Corporation were used for our simulation. The $B-H$ and $\tau_{y}-H$ curves were obtained by applying the least-squares curve fitting. $H$ is the magnetic flux intensity and $\tau_{y}$ is the yield stress of the MR fluid. The corresponding polynomials are described by the following equations:

$$
\begin{aligned}
H= & -102.64 B^{4}+324.64 B^{3} \\
& -91.47 B^{2}+180.01 B-0.17 \\
\tau_{y}= & 1.39 \times 10^{-8} H^{4}-7.41 \times 10^{-6} H^{3} \\
& +5.06 \times 10^{-4} H^{2}+0.31 H-0.40
\end{aligned}
$$

The next step was to calculate $H$ and $\tau_{y}$ from the Eq. (1) and (2) respectively. Then the pressure drop and the dynamic range of the valve were calculated using the following equations [14], [11]: 


$$
\begin{aligned}
\Delta P & =\Delta P_{\tau}+\Delta P_{\eta} \\
& =\frac{2 \cdot c \cdot \tau_{y}(H) \cdot\left(r_{e}-r_{i}\right)}{g}+\frac{12 \cdot \eta \cdot Q}{\pi \cdot g^{3}} \ln \left(\frac{r_{e}}{r_{i}}\right) \\
D & =\frac{\Delta P_{\tau}}{\Delta P_{\eta}}=5
\end{aligned}
$$

where $\Delta P$ is the total pressure drop in the valve, $\Delta P_{\tau}$ is the yield stress dependent pressure drop, $\Delta P_{\eta}$ is the viscosity dependent pressure drop and $D$ is the dynamic range, $\eta$ is the viscosity of MRF132DG, obtained from its datasheet and $Q$ is the flow rate of the MR fluid. $D$ is a figure of merit that indicates the operation range of the valve, ranging from free flow to a no flow. It is set to 5 in this case, leading to an approximation of $\mathrm{c}$ to 2.3 [11].

The volume $V$ of the valve is calculated as:

$$
V=\pi \cdot r_{o}^{2} \cdot h_{t}
$$

The power consumption $P$ of the valve is calculated as:

$$
P=i \cdot R_{c}^{2}
$$

where $i$ is the current of the coil and $R_{c}$ is the resistance of the coil. The wire diameter is $0.321 \mathrm{~mm}$ and the fill factor of the coil is $50 \%$.

Other parameters useful for the evaluation of our final optimal design is the reactivity $T_{v}$ and the response time $t$ of the MR valve. The reactivity is defined as the pressure drop reached in a certain amount of time, while the time response is defined as the time required to reach the desired magnetic field and, thus, the desired pressure drop. The reactivity and the time response are calculated as follows [15]:

$$
\begin{aligned}
& T_{v}=\frac{\Delta P_{\tau}}{\tau_{c}}=\frac{\Delta P_{\tau} \cdot i \cdot R_{c}}{N \cdot \phi} \\
& t=\frac{\Delta P_{\text {desired }}}{T_{v}}
\end{aligned}
$$

In Eq. 7, $\tau_{c}$ is the coil time constant, $N$ is the number of turns of the coil and $\phi$ is the magnetic flux.

\section{Optimization}

The DoE analysis in the software Minitab 19 was performed in order to create the experimental matrix with all the combinations of the previous factors. For each combination of factors, the magnetic flux density in the gap, the pressure drop, the power consumption and the volume were calculated using the equations of the previous section. The optimal combinations of factors was selected with DoE and the finite element analysis was performed at these points [16]. The next step was to deploy the finite element analysis results in order to approximate a response function, which can precisely model the magnetic flux density across the gap. This is an analytical polynomial function, fitted to the results by using RSM. In the end of the RSM process, the optimal levels of the factors and the response functions were obtained [17].

\section{RESULTS \& DISCUSSION}

The main effect plots provide us with a rough idea of the significance of the effect of each design factor on the two optimization objectives, the total pressure drop and the power consumption of each radial valve. The more horizontal the plot is, the less significant is the effect of the factor on the response [18]. It is evident from Fig. 5, that (a) only the length $g$ of the air gap affects significantly the total pressure drop $\Delta P$ in the valve and that (b) firstly, the current density $J$ and, secondly, the radius of the inner core $r_{e}$ present an important effect on the power consumption.

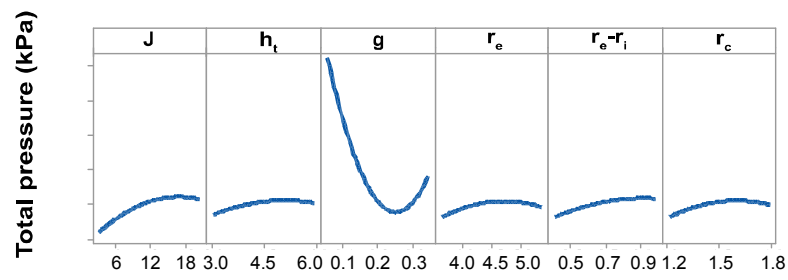

(a)

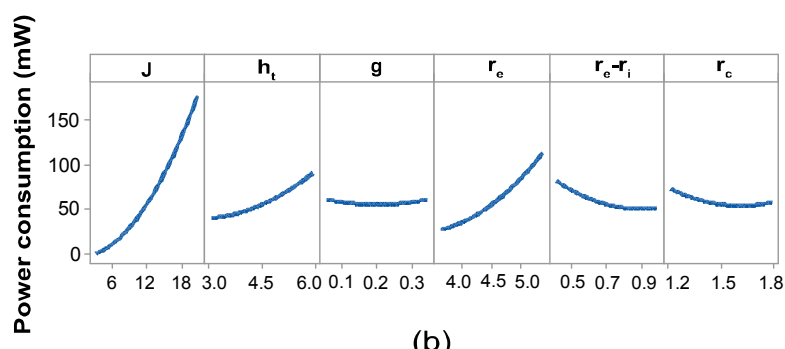

(b)

Fig. 5. Main effects graph of the different factors on (a) pressure drop and (b) power consumption of the valve.

In the final step of the RSM, the optimal dimensions of the valve were acquired with Minitab 19 and the optimal responses were calculated. Table II presents the optimal radial model parameters and compares them with an existing, not optimized radial valve prototype, fabricated based on previous studies [19]. In parallel, it shows, also, a comparison with the optimal annular valve model of our previous study [11] as well as with an annular valve prototype [19], in order to distinguish which configuration is more suitable for our wearable medical solution. In fact, the radial optimized model presents better characteristics compared to its corresponding prototype with regards to total volume, total pressure drop, flow rate, reactivity and power consumption, reducing total power consumption almost to half. On the other hand, it appears to have higher volume compared to the annular model, but yet acceptable for the wearable device requirements. Nevertheless, the total pressure drop achieved is higher and the power consumtion is lower $(37.73 \mathrm{~mW})$ compared to the annular optimized model (48.04 $\mathrm{mW})$.

Last but not least, the optimal geometric and current density parameters were used in order to create the final finite element model of the optimal radial valve. Fig. 6 depicts graphically the magnetic flux density and magnetic flux distribution on a $2 \mathrm{D}$ axis-symmetric radial valve model as well as specifically in the gap area, in 
order to validate their match with the magnetic flux values acquired by the analytical formulas.

TABLE II

ANNULAR AND RADIAL MODEL COMPARISON

\begin{tabular}{|c|c|c|c|c|}
\hline \multirow[b]{2}{*}{ Item } & \multicolumn{2}{|c|}{ Annular model } & \multicolumn{2}{|c|}{ Radial model } \\
\hline & Prototype & $\begin{array}{l}\text { Optimal } \\
\text { Model }\end{array}$ & Prototype & $\begin{array}{l}\text { Optimal } \\
\text { Model }\end{array}$ \\
\hline Outer radius $\mathrm{r}_{\mathrm{o}}(\mathrm{mm})$ & \multicolumn{2}{|c|}{5} & \multicolumn{2}{|c|}{5.5} \\
\hline Total height $\mathrm{h}_{\mathrm{t}}(\mathrm{mm})$ & 4.3 & 3.0 & 4 & 3.5 \\
\hline Air gap g $(\mathrm{mm})$ & 0.1 & 0.1 & 0.08 & 0.1 \\
\hline $\begin{array}{l}\text { Iron teeth's height } \mathrm{h} \\
(\mathrm{mm})\end{array}$ & 1 & 0.75 & 1 & 0.875 \\
\hline Wire diameter $\mathrm{D}_{\mathrm{w}}(\mathrm{mm})$ & \multicolumn{4}{|c|}{0.321} \\
\hline Fill factor $(\%)$ & 70 & 50 & 70 & 50 \\
\hline $\begin{array}{l}\text { Current density } J \\
\left(\mathrm{~A} / \mathrm{mm}^{2}\right)\end{array}$ & 11116 & 10.92 & 6 & 14.14 \\
\hline Total volume $\mathrm{V}\left(\mathrm{mm}^{3}\right)$ & 337.70 & 235.62 & 380.10 & 332.60 \\
\hline Current $(\mathrm{A})$ & - & 0.726 & - & 0.796 \\
\hline Flux density $\mathrm{B}_{\mathrm{g}}(\mathrm{T})$ & 0.339 & 0.348 & 0.274 & 0.316 \\
\hline Total pressure $\Delta \mathrm{P}(\mathrm{kPa})$ & 1050.20 & 812.43 & 723.14 & 881.0 \\
\hline Flow rate $\mathrm{Q}\left(\mathrm{cm}^{3} / \mathrm{s}\right)$ & 2.19 & 2.15 & 1.06 & 1.69 \\
\hline $\begin{array}{l}\text { Power consumption } \\
(\mathrm{mW})\end{array}$ & 109 & 48.04 & 63 & 37.72 \\
\hline Reactivity (MPa/s) & 9.46 & 11.03 & 3.12 & 8.56 \\
\hline $\begin{array}{l}\text { Response time for } 400 \\
\mathrm{kPa}(\mathrm{ms})\end{array}$ & - & 36.3 & - & 46.7 \\
\hline
\end{tabular}

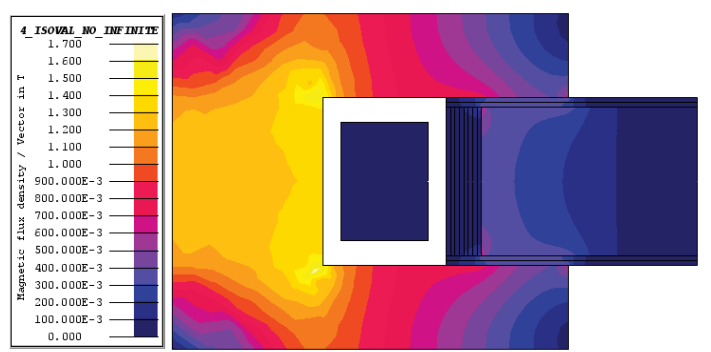

(a)

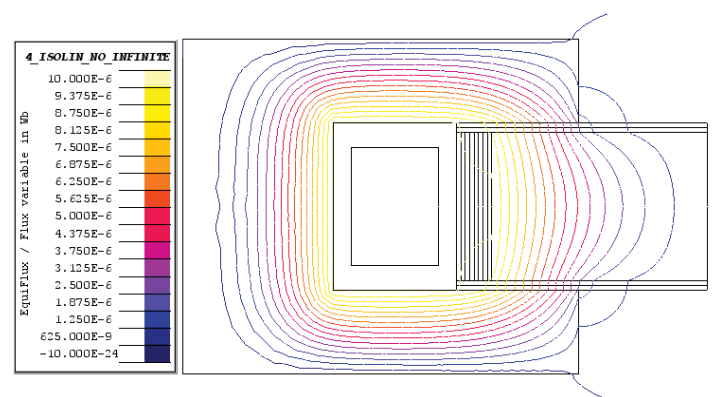

(b)

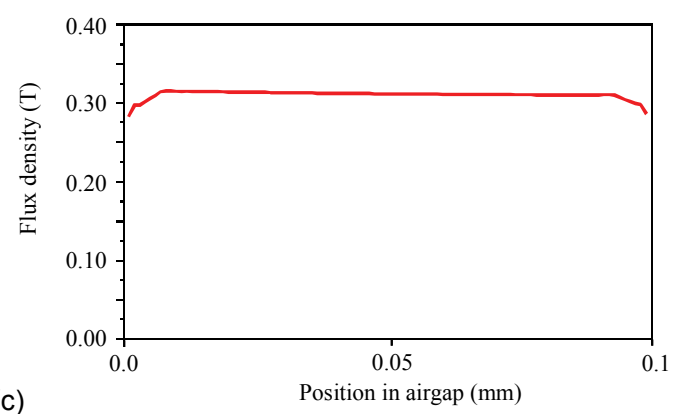

Fig. 6. Finite element analysis of (a) magnetic flux density and (b) magnetic flux distribution on the 2D axis-symmetric cross-section of the optimal radial MR valve. (c) Magnetic flux density distribution in the MR valve's airgap.

\section{CONCLUSIONS}

In this study we presented the optimization process and results of a radial miniature magnetorheological valve, aimed to be used in a wearable medical application for diabetic patients. Unlike previous studies, the valve is in a miniature form and it is deployed for the first time as pressure limiter for diabetic ulceration prevention. The objectives of the optimizations include the minimization of the valve's volume and power consumption, as well as the maximization of the total pressure drop of the valve, in order to meet the requirements of the wearable device. The paper includes a comparison with previously realized annular and radial valves' prototypes and with a previously optimized annular model. Indeed, the radial optimized model presents several positive characteristics compared to the annular one: lower volume, higher total pressure drop, higher flow rate and lower power consumption. All these make the radial optimized miniature valve a satisfying option for the pressure limiters of the wearable device.

\section{ACKNOWLEDGMENT}

The authors acknowledge support from the BRIDGE funding programme, conducted by the Swiss National Science Foundation (SNSF) and the Innosuisse-Swiss Innovation Agency.

\section{REFERENCES}

[1] M. R. Jolly, J. D. Carlson, and B. C. Munoz, "A model of the behavior of magnetorheological materials," in Smart Materials and Structures, vol.5, no. 5, pp. 607, 1996.

[2] A. Ghaffari, S. H. Hashemabadi, and M. Ashtiani, "A review on the simulation and modeling of magnetorheological fluids," in Journal of intelligent material systems and structures, vol. 26 , no. 8 , pp. 881904, 2015.

[3] Q.-H. Nguyen, S.-B. Choi, and N. M. Wereley, "Optimal design of magnetorheological valves via a finite element method considering control energy and a time constant," in Smart Materials and Structures, vol. 17, no. 2, pp. 025024 , 2008.

[4] A. Milecki and M. Hauke, "Application of magnetorheological fluid in industrial shock absorbers," in Mechanical Systems and Signal Processing, vol. 28, pp. 528-541, 2012.

[5] Y. Shiao and Q.-A. Nguyen, "Development of a multi-pole magnetorheological break," in Smart Materials and Structures, vol. 22, no. 6, pp. 065008, 2013.

[6] T.-H. Yang, H.-J. Kwon, S. S. Lee, J. An, J.-H. Koo, S.-Y. Kim, and D.-S. Kwon, "Development of a miniature tunable stiffness display using MR fluids for haptic application," in Sensors and Actuators A: Physical, vol. 163, no. 1, pp. 180-190, 2010.

[7] J.-H. Kim and J.-H. Oh, "Development of an above knee prosthesis using MR damper and leg simulator," in Proceedings 2001 ICRA. IEEE International Conference on Robotics and Automation (Cat. No. 01CH37164), vol. 4, IEEE, 2001, pp. 3686-3691.

[8] C.-W. Lung, E. T. Hsiao-Wecksler, S. Burns, F. Lin, and Y.-K. Jan, "Quantifying dynamic changes in plantar pressure gradient in diabetics with peripheral neuropathy," 
in Frontiers in bioengineering and biotechnology, vol. 4, pp. 54, 2016.

[9] A. Caselli, H. Pham, J. M. Giurini, D. G. Armstrong, and A. Veves, "The forefoot-to-rearfoot plantar pressure ratio is increased in severe diabetic neuropathy and can predict foot ulceration," in Diabetes care, vol. 25, no. 6, pp. 1066 1071, 2002.

[10] D. Grivon, Y. Civet, Z. Pataky, and Y. Perriard, "Design and characterization of a soft magneto-rheological miniature shock absorber for a controllable variable stiffness sole," in Archives of Electrical Engineering, vol. 64, no. 4, pp. 547-558, 2015.

[14] Lee, "Effects of internal stress concentrations in plantar soft-tissue - a preliminary three-dimensional finite element analysis," in Medical engineering \& physics, vol. 32, no. 4, pp. 324-331, 2010.

[15] D. Wang, H. Ai, and W. Liao, "A magnetorheological valve with both annular and radial fluid flow resistance gaps," in Smart Materials and Structures, vol. 18, no. 11, pp. 115001, 2009.

[16] D. Grivon, "Design, modelling and sensing possibilities of magneto-rheological based devices," EPFL, Tech. Rep., 2017, pp.49.
[11] S. L. Ntella, M.-T. Duong, Y. Civet, Z. Pataky and Y. Perriard, "Design optimization of miniature magnetorheological valves with self-sensing capabilities used for a wearable medical application," in 2020 IEEE/ASME International Conference on Advanced Intelligent Mechatronics (AIM). IEEE, 2020, pp. 409-414.

[12] N. Hayafune, Y. Hayafune, and H. Jacob, "Pressure and force distribution characteristics under the normal foot during the push-off phase in gait," in The foot, vol. 9, no. 2, pp. 88-92, 1999.

[13] W.-M. Chen, T. Lee, P. V.-S. Lee, J. W. Lee, and S.-J.

[17] D. C. Montgomery, Design and analysis of experiments. John Wiley \& Sons, 2017.

[18] A. Dean, D. Voss, and D. Dragulji, "Response surface methodology," in Design and analysis of experiments, pp. 565-614, Springer, 2017.

[19] J. Anthony, Design of experiments for engineers and scientists. Elsevier, 2014.

[20] D. Grivon, Y. Civet, Z. Pataky, and Y. Perriard, "Design and comparison of different magneto-rheological valves configurations," in 2015 IEEE International Conference on Advanced Intelligent Mechatronics (AIM). IEEE, 2015, pp. 818-823. 